Department of Cardiorheumatology, Kharkiv, Ukraine; ${ }^{3}$ V.N.Karazin Kharkiv Nataional University, Department of Pediatrics, Kharkiv, Ukraine

Background: The defeat of the cardiovascular system is considered a proven comorbid state in rheumatic diseases, including rheumatoid arthritis in adults. One of the markers of the cardiovascular failure formation is BNP, namely, its $\mathrm{N}$-terminal inactive fragment (NT-proBNP76), which accumulates in specific granules of cardiomyocytes. Its diagnostic value increases with the appearance of minimally expressed symptoms. The long-term course of JIA is also characterized by changes in the state of the cardiovascular system, and there may be no visible clinical manifestations. For their diagnosis a 6-minute walk test is widely used, including in children.

Objectives: To study the content of NT-proBNP in patients with juvenile idiopathic arthritis and compare with the level of exercise tolerance.

Methods: 10 patients with JIA (9 girls, 1 boy), average age $12.78 \pm 0.95$ years, were examined. All children had a polyarticular RF negative subtype of JIA with a disease duration of more than three years (average disease duration $69.56 \pm 17.07$ months), received basic methotrexate therapy and did not have dysfunction of the lower extremities joints. The control group included 7 healthy children, comparable by sex, average age $14.25 \pm 0.73$ years. An ECG, an ultrasound scan of the heart, and a 6-minute walk test (6MTX) were carried out with determining the distance traveled (6MWD) and the increase in heart rate. The level of the $\mathrm{N}$-terminal polypeptide of cerebral natriuretic hormone (B-type) (NT-proBNP) was determined in the morning, after waking up, and studied by competitive immunoassay on an IMMULITE 2000 analyzer ("Siemens").

Results: In children with JIA a decrease in myocardial contractility was not detected. Left ventricular ejection fraction $(62.17 \pm 0.83 \%(60.02-64.02)$ versus $69.84 \pm 0.85 \%(62.3-80.3), p<0.05)$ in children with JIA were within normal limits, but significantly lower than in the control group. According to the results 6MTX indicator 6MWD in JIA-patients was $490.51 \pm 11.40 \mathrm{~m}$ and in the control group $516.85 \pm 8.84 \mathrm{~m}(\mathrm{p}<0.05)$ and heart rate growth was 27.75 $\pm 2.30 \%$ versus in the control group $(37.38 \pm 3.86 \%), p<0.05$. A negative correlation between the increase in heart rate and the duration of the disease was found $(r=-0.7, p=0.05)$. The level of NT-proBNP in patients with JIA was within physiological values and amounted to $47.5 \pm 14.09 \mathrm{pg} / \mathrm{ml}(20-128 \mathrm{pg} /$ $\mathrm{ml})$, but this was higher than in children of the control group $(20.29 \pm 0.29 \mathrm{pg} /$ $\mathrm{ml}(20-22 \mathrm{pg} / \mathrm{ml}), \mathrm{p}<0.05)$. A high correlation was found between 6 MWD and NT-proBNP level $(r=0.8, p<0.03)$.

Conclusion: In children with JIA there is a decrease of the exercise tolerance that increases with the duration of JIA on the background of preserved myocardial contractility. This is accompanied by a higher basal NT-proBNP level than in healthy children.

Disclosure of Interests: None declared

DOI: 10.1136/annrheumdis-2020-eular.5926

\section{AB0987 1 STUDY ON THE FACTORS AFFECTING THE FINAL ADULT HEIGHT IN JUVENILE IDIOPATHIC ARTHRITIS}

C. Hsin-Yu ${ }^{1}$, H. Ya-Chiao ${ }^{1}$, B. L. Chiang ${ }^{1} .{ }^{1}$ National Taiwan University Hospital, Taipei, Taiwan, Republic of China

Background: Juvenile idiopathic arthritis (JIA), a chronic inflammatory disease involving limited joints and/or constitutional symptoms in childhood or adolescent, might affect body height and result in short stature in adulthood. Well-controlled disease activity is beneficial in improving growth impairment in JIA patient. Objectives: To identify any factors that influence final adult height in the patient with juvenile idiopathic arthritis.

Methods: We retrospectively reviewed the medical records of JIA patients between 2009 to 2019 in National Taiwan University Hospital. The diagnosis of JIA was according to the International League of Associations for Rheumatology (ILAR) criteria. Personal history, laboratory reports, and medication were analyzed. The difference between final adult height and target height was calculated in each patient. We defined whose final adult height higher than target height as positive group and the others as negative group. A cox univariate proportional hazards model was applied to compare the variables between these two groups.

Results: Total 120 patients are collected. There are $74(61.7 \%)$ and 46 (38.3\%) cases in the positive and the negative group, respectively. The mean onset age of disease is 11.783 .78 in positive group and 10.834 .04 in negative group. Male is more than female in both groups, with a ratio of $1.7: 1$ and $2.7: 1$ respectively. Enthesis-related arthritis accounted for the most in positive group, however, in negative group, oligo-arthritis was the most common type. There are slightly more patients having received biologics in positive group than negative group without significance $(64.8 \%$ vs. $54.3 \%, p=0.33)$. Among the patients treated with etanercept, those in the negative group had a significantly younger age when they started the treatment compare to the positive group (11.83.1 vs. 13.9 3.1, $P=0.01$ ). The patients in positive group had significantly higher final adult height (171.08.7 vs. $162.98 .1, P<0.001)$. The univariate analysis showed that the age when etanercept treatment started was associated with the occurrence of a negative difference between final height and target height (odds ratio $=0.80$ $95 \% \mathrm{Cl}=0.67-0.96, P=0.02)$.

Conclusion: In JIA patients, $38.3 \%$ of them had a negative difference between final adult height and target height. The subtype might play a critical role in affecting the growth of patients. A younger age when the child received etanercept was associated with a lower attained adult height than target height, however, it needs further analysis on the medication of these children.

\section{References:}

[1] S.-J. Wang et al., Attained Adult Height in Juvenile Rheumatoid Arthritis with or without Corticosteroid Treatment, Clin Rheumatol (2002) 21:363-368

[2] P Tynja"la et al., Impact of anti-TNF treatment on growth in severe juvenile idiopathic arthritis, Ann Rheum Dis 2006;65:1044-1049

[3] Edward H. Giannini et al., Effects of Long-Term Etanercept Treatment on Growth in Children With Selected Categories of Juvenile Idiopathic Arthritis, ARTHRITIS \& RHEUMATISM Vol. 62, No. 11, November 2010, pp 3259-3264

[4] Ashley Shafferman et al, Changes in Body Mass Index in Children with Juvenile Idiopathic Arthritis Treated with Tumor Necrosis Factor Inhibitors, Rheumatol 2014;41;113-118

[5] Lianne Kearsley-Fleet et al., Growth in children and adolescents with juvenile idiopathic arthritis over 2 years of treatment with etanercept: results from the British Society for Paediatric and Adolescent Rheumatology Etanercept Cohort Study, Rheumatology 2015;54:1279-1285

[6] Florence Uettwiller et al., Effect of Biologic Treatments on Growth in Children with Juvenile Idiopathic Arthritis, J Rheumatol 2014;41;128-135

[7] Lianne Kearsley-Fleet et al., Short-term outcomes in patients with systemic juvenile idiopathic arthritis treated with either tocilizumab or anakinra, Rheumatology 2019;58:94-102

Disclosure of Interests: None declared

DOI: 10.1136/annrheumdis-2020-eular.1654

\section{AB0988 \\ CLINICAL FEATURES AND ANALYSIS OF MEFV GENE IN 31 PATIENTS WITH FAMILIAL MEDITERRANEAN FEVER (FMF)}

T. Ishizuka ${ }^{1}$, K. Fujioka ${ }^{1}$, M. Tangiku ${ }^{1}$, S. Inui ${ }^{2}$, H. Tani ${ }^{1}$, A. Miwa ${ }^{1}$, T. Ikeda ${ }^{2}$, K. Taguchi ${ }^{2}$, H. Morita ${ }^{1,2}$, T. Toma $^{3}$, A. Yachie ${ }^{3}$. ${ }^{1}$ Gifu Municipal Hospital, Center of General Internal Medicine and Rheumatology, Gifu, Japan; ${ }^{2}$ Gifu University Graduate School of Medicine, Department of General Internal Medicine, Gifu, Japan; ${ }^{3}$ Kanazawa University Graduate School of Medicine, Kanazawa, Japan

Background: FMF is recessive systemic autoinflammatory disorder characterized by recurrent fever, peritonitis, pleuritis, pericarditis and arthritis accompanied with headache and abdominal pain. Mutation of MEFV gene encoding pyrin resulted in inflammasome activation and the uncontrolled production of IL-1 $\beta$. Overview of pathogenesis, clinical features and management in Japanese patients with FMF had been reported. However, the differences of clinical features between mutated and non-mutated of MEFV still remain unclear.

Objectives: We have analyzed 31 Japanese patients with FMF in Gifu district to clarify the association between various clinical features and mutation of MEFV. Methods: Genomic DNA were purified from white blood cells in 31 FMF patients, and mutated MEFV has been explored. We have analyzed MEFV, TNFRSF1A, MVK and NLRP3 genes in 31 patients with FMF except for 1 patient. Therefore, we excluded another autoinflammatory diseases such as TNF receptor-associated syndrome (TRAPS), mevalonate kinase deficiency and cryopyrin-associated periodic syndrome. Clinical symptoms and laboratory data were analyzed around onset time. Each patient had been treated with colchicine $(0.5-2 \mathrm{mg})$.

Results: Characteristics of Patients with FMF (22 female/9 male) were as follows; Onset time were $0-56$ years-old $(21.4 \pm 11.8)$, and Frequencies of clinical symptoms such as periodic fever, headache, abdominal pain, arthralgia, chest pain, cervical lymph nodes swelling, and myalgia were $31 / 31$, $9 / 31,8 / 31,6 / 31,5 / 31,3 / 31$ and $1 / 31$, respectively (double symptoms were observed). Patients with FMF were divided into 3 groups as follows; Patients with typical compound heterozygous mutations of MEFV (E148Q /M694I) which indicated exon 10 mutation, were 5 cases (G1). Patients with atypical mutations, except for exon 10, such as $133 \mathrm{G}>\mathrm{A}$ in $3 \mathrm{UTTR}$, exon 1 (E84K), 2 (L110P, E148Q), 3(R202Q, P257L, G304R, P369S, R408Q), 5(S503C) and $9(I 591 \mathrm{M})$ were 13 cases (G2). Patients with no mutations in MEFV gene were 12 cases (G3). There were no significant differences of age at first visiting hospital (FV)and onset age of fever attack (O) (FV: $29.0 \pm 15.6,27.1 \pm 12.5$ years-old (yo) and $34.7 \pm 12.7$ yo, $0: 21.0 \pm 17.6$ yo, $17.8 \pm 12.1$ yo and $25.2 \pm$ 6.5). But significant differences in duration of fever attack (D) and frequency of fever attack (FF) between $\mathrm{G} 1$ and $\mathrm{G} 2$ or G3 were observed as follows; (D: $2.2 \pm 0.4$ days vs $5.5 \pm 3.1$ days, $P<0.05$, and $3.8 \pm 1.7$ days), FF: $0.72 \pm 0.3$ / 